\title{
Proactive Traffic Information Control in Emergency Evacuation Network
}

\author{
Zhengfeng Huang ${ }^{1,2}$ \\ ${ }^{1}$ Faculty of Maritime and Transportation, Ningbo University, Ningbo 315211, China \\ ${ }^{2}$ School of Transportation, Southeast University, Nanjing 210096, China \\ Correspondence should be addressed to Zhengfeng Huang; huang321000@gmail.com
}

Received 2 April 2014; Accepted 24 September 2014

Academic Editor: Zbigniew Leśniak

Copyright (c) 2015 Zhengfeng Huang. This is an open access article distributed under the Creative Commons Attribution License, which permits unrestricted use, distribution, and reproduction in any medium, provided the original work is properly cited.

Traffic demand in emergency evacuation is usually too large to be effectively managed with reactive traffic information control methods. These methods adapt to the road traffic passively by publishing real-time information without consideration of the routing behavior feedback produced by evacuees. Other remedy measures have to be prepared in case of nonrecurring congestion under these methods. To use the network capacity fully to mitigate near-future evacuation traffic congestion, we propose proactive traffic information control (PTIC) model. Based on the mechanism between information and routing behavior feedback, this model can change the route choice of evacuees in advance by dissipating strategic traffic information. Generally, the near-future traffic condition is difficult to accurately predict because it is uncertain in evacuation. Assume that the value of traffic information obeys certain distribution within a range, and then real-time traffic information may reflect the most-likely near-future traffic condition. Unlike the real-time information, the proactive traffic information is a selection within the range to achieve a desired level of the road network performance index (total system travel time). In the aspect of the solution algorithm, differential equilibrium decomposed optimization (D-EDO) is proposed to compare with other heuristic methods. A field study on a road network around a large stadium is used to validate the PTIC.

\section{Introduction}

For the natural or man-made disasters, the surrounding road network should be fully utilized to evacuate the affected people. Otherwise, stochastic evacuation flows may induce nonrecurring traffic congestion. Therefore, optimizing the operation of evacuation flows to improve emergency evacuation efficiency is one of the main goals of the disaster relief. From the perspective of behavior, how to adjust evacuees' route-choice result to mitigate congestion in advance is a critical point. In other words, we should consider evacuees' routing behavior feedback in the evacuation management.

Compared to general transportation network management, routing behavior feedback is less considered in the evacuation condition. Unlike the widely applied cases in the former condition, such as street capacity optimization [1] and signal optimization [2], only few studies are found in the latter condition, that is, Xie and Turnquist [3] in the planning of lane reversals and intersection crossings mitigation, Chiu and Mirchandani [4], and Paz and Peeta [5] in the dynamic choice of information-publishing routes.

Traffic information control can be a feasible evacuation management. However, decision makers usually passively published real-time information to evacuees whose routechoice result is not being optimized. Thus, the performance index of evacuation network can be improved only to a limited extent. Generally, the real-time information can solely reflect the near-future traffic condition with the maximum probability because this condition is uncertain in evacuation. At the current time interval, the value of near-future traffic information may emerge to be a probabilistic distribution within a range. Selecting any of them may lead to varied route-choice results of evacuees; thus, we need to develop a method to choose the appropriate value instead of the real-time value as the information provision to improve evacuation efficiency. 
To design traffic information considering behavior feedback, the mapping between traffic information and evacuation flows should be addressed. Under specific information, the mapping is generated by two intermediate processes, the route choice and traffic loading. This is also the routing behavior feedback of evacuees, through which the decision maker can design the information to optimize the system performance. We define such optimization as proactive traffic information control (PTIC). Thus, this type of information optimization is akin to network design problem, which belongs to Stackelberg game. Because of the non-closedform of traffic-loading simulation steps involved in this application, the general operations research methods used for nonlinear planning cannot be applied. The useful methods are heuristic, such as equilibrium decomposed optimization algorithm [6], linear approximation algorithm [7], and intelligent algorithm [8]. The last algorithm can have the potency of global convergence because the searching process is akin to enumeration. However, it is imperfect for its low efficiency. The first method is a numerical method which uses onedimensional search to reduce the solution range, but the global solution may be ignored at the initial iterations with large step size and also we cannot ensure the convergence of the solutions in the whole operation. The main idea of the second method is to use Taylor expansion to change the objective to a linear function to solve drop direction and golden selection method to solve step size, such as FrankWolfe (F-W) algorithm. The only note is that the derivative or difference of objective with respect to decision variable should be provided in the generated subproblem. However, this method may converge to a local optimum near the initial solution because the drop direction and step size are too accurate to jump out of the local trap.

To better mitigate near-future congestion for emergency evacuation network, we propose PTIC to provide the traffic information strategically according to routing behavior feedback. The minimal total system travel time (TSTT) is set as the objective, which is composed of total evacuation time, arrival time penalty, and congestion cost. A corresponding differential equilibrium decomposition optimization (D-EDO) algorithm is proposed and demonstrated to be advantageous in the accuracy.

In what follows, the PTIC model and solving steps of DEDO are introduced. Then, the theoretical method is applied in the field case of evacuation network around Nanjing Olympic Stadium in China.

\section{Method}

2.1. PTIC Model. The following constraints (1-8) and the minimal TSTT objective (9) are designed to formulate PTIC model. The constraints are in accord with one-shot stochastic dynamic traffic assignment, composed of stochastic route choice and one-shot traffic loading. The reason for adopting a nonequilibrium model is that no historic travel experience can help evacuees make a game decision; the reason for adopting stochastic model is that evacuees cannot know the a priori flow condition in the nonrecurring event.
2.1.1. Route-Choice Utility. There are some exogenous routerelated variables influencing evacuees on the route choice. The determination of exogenous variables mainly depends on two criteria (evacuation time and information reliability). As for evacuation time, it is no doubt that people prefer to choose shorter routes. Besides, Wardman et al. [9] concluded, using a stated preference (SP) survey, that delay time is more useful than travel time in the route choice. With respect to information reliability, risk aversion will emerge in travelers' mind when unreliable information is provided. Hua et al. [10] showed that the disseminated delay-time reliability is a significant factor influencing route choice. Therefore, exogenous variables of route length, delay time, and disseminated delay-time reliability are chosen as the route-choice explanatory variables. For easy recognition, these three explanatory variables corresponding to route $r$ at an information-updating interval $\tau$ are represented as $L_{r}(\mathrm{~m})$, $D_{r}^{\tau}(\min )$, and $R_{r}^{\tau}(\%)$ in sequence. The utility value of route $r$ at the information-updating interval $\tau$ can be calculated by

$$
V_{r}^{\tau}=x+\beta_{1} \cdot L_{r}+\beta_{2} \cdot D_{r}^{\tau}+\beta_{3} \cdot R_{r}^{\tau}-C F_{r} .
$$

In formula (1), the evacuees are assumed to be homogeneous, so use constant $x$ to represent the utility value of endogenous attributes of evacuees. We can lose this term "commensurable and can be removed" in the discrete choice model. To consider the overlap line problem, the $C$-logit model [11], a type of multinomial logit model, is usually adopted as the discrete choice model because of its simple form and easy calibration. The variable $C F_{r}=$ $\beta_{0} \ln \sum_{c \in C_{r}} w_{c, r} n_{c, r}$ is the commonality factor in the utility of $C$-logit model. The variable $w_{c, r}$ is the proportional weight (often represented as a length ratio) of the overlap section $c$ between path $r$ and another route of the same OD pair. The set $C_{r}$ includes all the overlap sections related to route $r$. The variable $n_{c, r}$ is the number of routes, connecting the same OD pair with $r$, which share the overlap section $c$. The explanatory-variable coefficients $\beta_{1}, \beta_{2}, \beta_{3}$, and $\beta_{0}$ need to be calibrated using SP survey.

2.1.2. Analytical Route-Choice Probability Function. According to the $C$-logit model, the analytical route-choice probability for choosing route $r$ from origin cell $i$ to destination at an information-updating interval $\tau$ can be determined by

$$
p_{i, r}^{\tau}=\frac{\exp \left(V_{r}^{\tau}\right)}{\sum_{h \in I_{i}} \exp \left(V_{h}^{\tau}\right)}, \quad \forall r \in I_{i},
$$

where $I_{i}$ is the route set from cell $i$ to destination. Because the destination of evacuation network is normally set as a virtual node, the index for destination can be eliminated to reduce notation complexity. The destination is known implicitly through the route index.

2.1.3. One-Shot Traffic Loading. Daganzo's [12] cell transmission model (CTM) can load traffic dynamically and successfully embedded in simulation software VISTA. But this basic cell cannot extract congestion information. In the following, we propose an enhanced CTM to overcome 
this shortcoming. The following variables at each (loading) interval are defined a priori:

$\Gamma(i)$ : set of cells succeeding cell $i$,

$\Gamma^{-1}(i)$ : set of cells preceding cell $i$,

$Q_{i}$ : maximal number of vehicles that can flow into or out of cell $i$ at interval $t$,

$N_{i}$ : maximal number of vehicles that can be present inside a cell $i$ at interval $t$,

$g_{i}^{t}$ : demand from source cell $i$ at interval $t$,

$\mu_{i}^{t}$ : vehicle occupancy of cell $i$ at interval $t$,

$\omega_{i}^{t}$ : flow outflowing from cell $i$ at interval $t$,

$\omega_{i, j}^{t}$ : flow from cell $i$ to cell $j$ at interval $t$.

It is noted that the unit time interval for traffic loading is shorter than the information-updating time interval.

Assuming the time-varying demands and route-choice ratios are provided, we can turn to the procedure of the enhanced CTM (shown in Figure 1) to simulate the flow. Regarding the proposed varied length cell, each link in our model is divided into three types of cells composed of 1 tail cell, 1 head cell, and a few approach cells (its number is based on the number of stream directions). Head cell and tail cell are used to capture the intersection queue length and traffic spillback separately. The latter two indexes could serve the representation of congestion cost in the PTIC objective. For example, we can let the length of approach cell be equal to unit time first and set the length of head cell to be equal to a threshold value required by decision maker to penalize the intersection queue and give the remaining length to tail cell to check the queue spillback for penalization.

Assuming backward propagation owns free-flow speed, the following procedure can be used to simulate vehicle occupancy $\mu_{i}^{t+1}$ of cell $i$ for interval $t+1$, based on previous vehicle occupancies ( $\mu_{i}^{1}$ is set to be 0 ).

(1) Formulations for Sending and Receiving Flows. The formulae for receiving and sending flows of cell $i$ can be described in

$$
\begin{aligned}
& X_{i}^{t}=\min \left\{Q_{i}, \frac{N_{i}}{l_{i}}, \mu_{i}^{t-l_{i}+1}-\sum_{m=t-l_{i}+1}^{t-1} \omega_{i}^{m}\right\}, \\
& Y_{i}^{t}=\min \left\{Q_{i}, \frac{N_{i}}{l_{i}}, N_{i}-\mu_{i}^{t}\right\},
\end{aligned}
$$

where $l_{i}$ is the needed number of time intervals used to pass through cell $i$ with the free-flow speed. It depends on the type that cell $i$ belongs to.

(2) Transmission Flow Formulation. We assume cell $i$ is a tail cell here. The transmission flow formula for flow from tail cell $i$ to head cell $j$ is expressed in

$$
\omega_{i, j}^{t}=\min \left\{X_{i}^{t}, Y_{j}^{t}\right\}, \quad \forall j \in \Gamma(i) .
$$

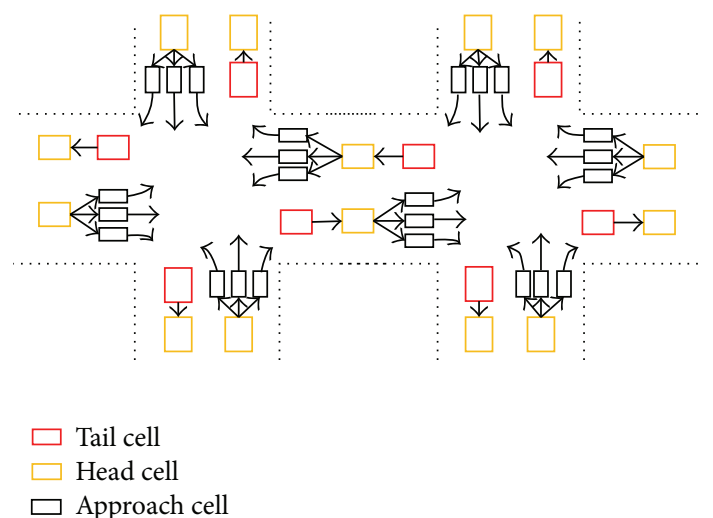

FIGURE 1: Enhanced CTM.

The transmission flow formula for flow from head cell $j$ to approach cell $k$ is denoted by formula (5). $\eta_{j, k}^{t}$ is the proportions of vehicle occupancy $\mu_{j}^{t}$ that can go to cell $k$. It is an implicit function with respect to route-choice probability $p_{i, r}^{\tau}$. If $\eta_{j, k}^{t}$ equals zero then the corresponding term inside the brace of formula (5) should be deleted:

$$
\begin{gathered}
\omega_{j}^{t}=\min \left\{X_{j}^{t}, \min \left\{\frac{Y_{k}^{t}}{\eta_{j, k}^{t}} \mid k \in \Gamma(j)\right\}\right\} \\
\omega_{j, k}^{t}=\eta_{j, k}^{t} \omega_{j}^{t}, \quad \forall k \in \Gamma(j) .
\end{gathered}
$$

The transmission flow formula for flow from approach cell $k$ to tail cell $i$ is denoted by

$$
\begin{array}{r}
\omega_{k, i}^{t}=X_{k}^{t}, \quad \forall k \in \Gamma^{-1}(i) \mid Y_{i}^{t} \geq \sum_{j \in \Gamma^{-1}(i)} X_{j}^{t}, \\
\omega_{k, i}^{t}=\operatorname{mid}\left\{X_{k}^{t}, Y_{i}^{t}+X_{k}^{t}-\sum_{j \in \Gamma^{-1}(i)} X_{j}^{t}, \gamma_{k, i}^{t} Y_{i}^{t}\right\}, \\
\forall k \in \Gamma^{-1}(i) \mid Y_{i}^{t}<\sum_{j \in \Gamma^{-1}(i)} X_{j}^{t},
\end{array}
$$

where $\gamma_{k, i}^{t}$ equals $X_{k}^{t} / \sum_{h \in \Gamma^{-1}(i)} X_{h}^{t}$ which represents the fraction of sending flow $X_{k}^{t}$ in all the sending flows at preceding cells of cell $i$ at interval $t$.

During each time interval, both transmission flows in formulae (5) and (6) should be rounded to ensure that the flows and vehicle occupancies are integers.

(3) Vehicle Occupancy Formulation. The following formula is used to update the vehicle occupancy of cell $i$ for the next interval:

Origin cell $i: \mu_{i}^{t+1}=g_{i}^{t}+\mu_{i}^{t}-\omega_{i}^{t}$,

Nonorigin and nondestination cell $i$ :

$$
\mu_{i}^{t+1}=\mu_{i}^{t}+\sum_{k \in \Gamma^{-1}(i)} \omega_{k, i}^{t}-\omega_{i}^{t} .
$$


2.1.4. Objective Formulation. The objective is designed in formula (8) and is composed of three terms. The first term is the total evacuation time. In the enhanced CTM, the total evacuation time is equal to total vehicle occupancies during the evacuation when veh time interval is the unit. The second term is the arrival time penalty, which means each consumed time interval should be penalized once the vehicle departs the origin. Why is penalty added to the cost of vehicles according to its arriving time at destination? One reason is to quicken the step of emergency. Another advantage is that the earlier leaving time will be chosen in advance when two routes with the same cost of different departure time need to be compared. The third term is the congestion cost, which penalizes the consumed time in the long queue and spillback:

$$
\begin{aligned}
& \min Z(\mathbf{y}) \\
& =\sum_{i \in I, t \in T} \mu_{i}^{t}(\mathbf{y})+\widetilde{M} \cdot\left\{\sum_{i=D, t \in T}\left(\mu_{i}^{t}(\mathbf{y}) \cdot t\right)-\sum_{i \in O, t \in T}\left(g_{i}^{t} \cdot t\right)\right\} \\
& \quad+\sum_{i \in I^{\prime}, t \in T} M_{i} \cdot \varphi_{i}^{t}(\mathbf{y}) \cdot \mu_{i}^{t}(\mathbf{y})
\end{aligned}
$$

where $I$ is the set of all the cells, $I^{\prime}$ is the set of all the head and tail cells, $O$ is the set of origin cells, and $D$ is the dummy destination cell. $T$ is the set of all time intervals and $\widetilde{M}$ and $M_{i}$ are the penalties for the arrival time and oversaturated vehicles separately. The traffic state judgment of cell $i$ at time interval $t$ can roughly be the following formula:

$$
\varphi_{i}^{t}(\mathbf{y})= \begin{cases}1 & \text { if }\left(N_{i}-Q_{i} \leq \mu_{i}^{t}(\mathbf{y}) \leq N_{i}\right) \\ 0 & \text { o.w. }\end{cases}
$$

It is obvious that if long queue or queue spillback occurs at cell $i$, corresponding penalty $M_{i}$ is added to decrease the dynamic routes and their vehicles passing through this cell at this time.

The vector $\mathbf{y}$ in formula (8) represents the decision variable vector composed of information variables shown in formula (1). In the proactive traffic information model, y can affect the objective indirectly. Its influence process is shown as follows: information variables $\rightarrow$ analytical routechoice probability $\rightarrow$ simulated vehicle occupancy $\rightarrow$ TSTT. To guarantee the mapping between the information and the evacuation flows is unique, we set single decision variable at each origin point per information-updating interval. The problem about how to determine decision variables from the selected information should be solved according to the specific application.

2.2. Solving Steps of D-EDO. The logical framework of solving PTIC in emergency evacuation is shown in Figure 2. The inputs are the physical attributes of the evacuation network, time-varying evacuation demand, the explanatory-variable coefficients of route-choice utility, and the time-varying information boundary values. The output is the published strategic information. Two main modules (one-shot stochastic dynamic traffic assignment and D-EDO procedure) shown in Figure 2 are elaborated in the following subsections.
One-shot stochastic dynamic traffic assignment module is actually within the module of D-EDO algorithm. It is composed of stochastic route choice and dynamic traffic loading. The updated information of D-EDO should be the preparedness of utility, which can immediately be the input of this module. At each information-updating interval of the internal process, $C$-logit function is applied to obtain the analytical route-choice ratio; and then a one-shot CTM simulation is performed. Step by step, traffic loadings at the following information-updating intervals are calculated. This module provides the data for the corresponding objective calculation of the D-EDO algorithm.

The D-EDO is proposed by combining the EDO and F$\mathrm{W}$ algorithms. The EDO is set as the outside layer algorithm. For every certain number (equal to the decision variable number) of iterations, we would choose the current minimal boundary solution of EDO as the initial input of F-W. We could solve its surrounding local optimum by the inside layer F-W algorithm then. When the algorithm of EDO is over, we compare all these local solutions to obtain the minimal result as the optimum. The EDO can go through diverse feasible solutions as they are narrowed gradually from the initial maximal boundary range. The effectiveness of F-W is related to initial input. The global optimum is more likely to be found using F-W with diverse initial inputs than uniform ones. Therefore, the advantages of both EDO and F-W are utilized to obtain a more accurate result which is expected to avoid the local optima. In the drop direction searching process of F-W, difference instead of deviation is prepared because the non-closed-form of the problem (due to the embedding of the simulation process) prohibits the solving of the deviation. Therefore, we name this method as differential decomposed equilibrium optimization method.

The steps of D-EDO are as follows.

Step 1 (initialization). Select vectors $\mathbf{L}=(\ldots, L(i, \tau), \ldots)$ and $\mathbf{U}=(\ldots, U(i, \tau), \ldots)$ representing, respectively, the initial lower and upper bounds on information variable vector, where $i$ is the origin cell and $\tau$ is the information-updating time interval. Simulate the vehicle occupancy vectors $\boldsymbol{\mu}(\mathrm{L})$ and $\boldsymbol{\mu}(\mathbf{U})$. Evaluate the objective value for each vector, $Z(\mathbf{L})$ and $Z(\mathbf{U})$. Set $\delta=0$.

Step 2 (F-W algorithm).

Step 2.1. Set $\delta=\delta+1$. Let $\Phi=\operatorname{argmin}_{\mathbf{L}, \mathbf{U}}\{Z(\mathbf{L}), Z(\mathbf{U})\}$ be the feasible solution.

Step 2.2. Calculate the difference of $\mu(\Phi)$ (and $\varphi(\Phi) \cdot \mu(\Phi)$ ) with respect to $\Phi$ through flow simulation; obtain the form $\nabla \mu(\boldsymbol{\Phi})$ (and $\nabla[\varphi(\boldsymbol{\Phi}) \cdot \mu(\boldsymbol{\Phi})])$. Find auxiliary feasible solution $\Psi$ such that the direction from $\Phi$ to $\Psi$ provides the maximum feasible drop in the following linearization, which has already deleted the constant terms in the initial objective function:

$$
\begin{aligned}
\min _{\Psi} \sum_{j \in O, \tau=\widetilde{T}}\left\{\sum_{i \in I, t=T} \nabla \mu_{i}^{t}(\phi(j, \tau))\right. \\
+\widetilde{M} \cdot \sum_{i \in D, t \in T}\left(\nabla \mu_{i}^{t}(\phi(j, \tau)) \cdot t\right)
\end{aligned}
$$




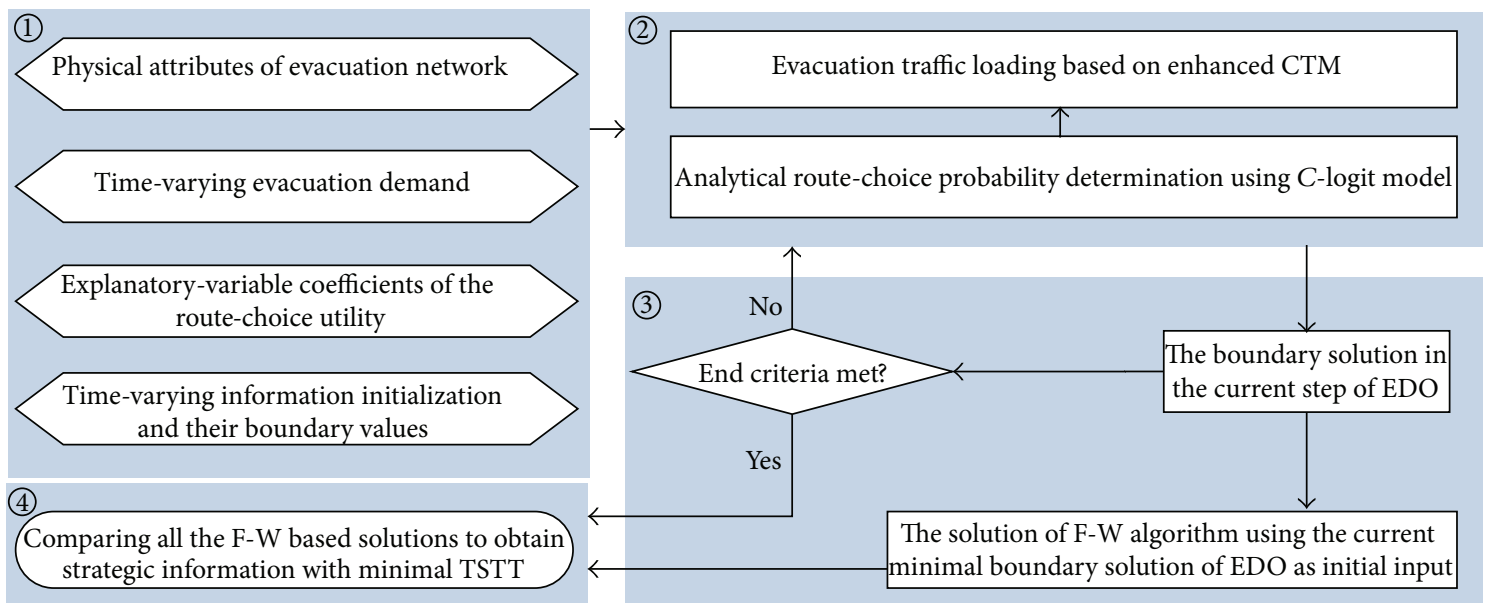

(1) Input

(2) One-shot stochastic dynamic traffic assignment

(3) $\mathrm{D}$-EDO procedure

(4) Output

FIgURE 2: Logical framework of solving PTIC.

$$
\begin{aligned}
& \left.+\sum_{i \in I^{\prime}, t \in T}\left(M_{i} \cdot \nabla\left[\varphi_{i}^{t}(\phi(j, \tau)) \cdot \mu_{i}^{t}(\phi(j, \tau))\right]\right)\right\} \\
& \cdot \psi(j, \tau),
\end{aligned}
$$

where $\widetilde{T}$ is the set of information-updating intervals and $\phi(j, \tau)$ and $\psi(j, \tau)$ are the elements in $\boldsymbol{\Phi}$ and $\boldsymbol{\Psi}$.

Step 2.3. Use golden selection method to find the associated optimal step size $\lambda$ such that

$$
\min _{\lambda} Z_{\delta}(\Phi+\lambda \cdot(\Psi-\Phi)),
$$

and update the current solution by $\boldsymbol{\Phi}=\boldsymbol{\Phi}+\lambda(\boldsymbol{\Psi}-\boldsymbol{\Phi})$.

Step 2.4. If the gap between two successive solutions is less than $\varepsilon$, then set $\widetilde{Z}_{\delta}=Z_{\delta}$ and turn to Step 3 to continue the algorithm of EDO; otherwise, turn back to Step 2.2.

Step 3 (golden selection). For each $i$ and $\tau$, the search procedure specifies the following two points in the interval $[L(i, \tau), U(i, \tau)]:$

$$
\begin{aligned}
& \rho(i, \tau)=U(i, \tau)+0.618(L(i, \tau)-U(i, \tau)), \\
& \pi(i, \tau)=L(i, \tau)+0.618(U(i, \tau)-L(i, \tau)) .
\end{aligned}
$$

Let other elements in $\mathbf{L}$ (and $\mathbf{U}$ ) be assigned to other elements in $\boldsymbol{\rho}$ (and $\pi$ ). Perform flow simulation to obtain the vehicle occupancy vectors $\boldsymbol{\mu}(\boldsymbol{\rho})$ and $\boldsymbol{\mu}(\boldsymbol{\pi})$. Evaluate the objective value for each vector, $Z(\boldsymbol{\rho})$ and $Z(\boldsymbol{\pi})$. If $Z(\boldsymbol{\rho}) \leq Z(\boldsymbol{\pi})$, then let $\mathbf{U}=\boldsymbol{\pi}$; otherwise, let $\mathbf{L}=\boldsymbol{\rho}$.

Step 4 (stopping criterion). If $|\mathbf{U}-\mathbf{L}| \leq \varepsilon$, output optimized objective $\min \left\{\widetilde{Z}_{\chi}, \forall \chi=1,2, \ldots, \delta\right\}$ and the corresponding solution y; otherwise, turn back to Step 2.

\section{Method Application}

3.1. Input. Input the evacuation network, evacuation demand, analytical route-choice probability function, and initialized information in sequence.

3.1.1. Evacuation Network and Demand. The Nanjing Olympic Center (Figure 3(a)) in China, with a capacity of 87,000 people, is assumed to be under the threat of a terrorist incident. People in the stadium should evacuate the stadium and nearby parking lots. They are not safe until they have escaped the described road network that surrounds the Olympic Stadium. The roads within the network constitute a closed area that allows only a northeast bound flow. Parking lots, located on the north side, south side, and east side (two lots on the east side) of the Olympic Center, are the origins for the evacuation. The VMSs are set up at origins $O_{1}$ and $\mathrm{O}_{2}$. The cell structure containing a dummy destination (with unlimited maximal vehicle occupancy and flow capacity) is shown in Figure 3(b). Its corresponding evacuation traffic demand and cell characteristics are shown in Table 1.

\subsubsection{Analytical Route-Choice Probability Function and Infor-} mation Initialization. Considering the limitation of evacuees' information analysis capability, at most three routes are assumed available for travelers to choose from at each origin cell. These chosen routes $(K \leq 3)$ are space-constrained candidate routes in this study. The comparison factors are route length, number of intersections, number of lanes, and number of left turns, in that order. The routes with lower factor values are more likely to be chosen for the set.

The candidate route sets of the origin cells are listed in Table 2 . The third column shows the resolved commonality factor. 


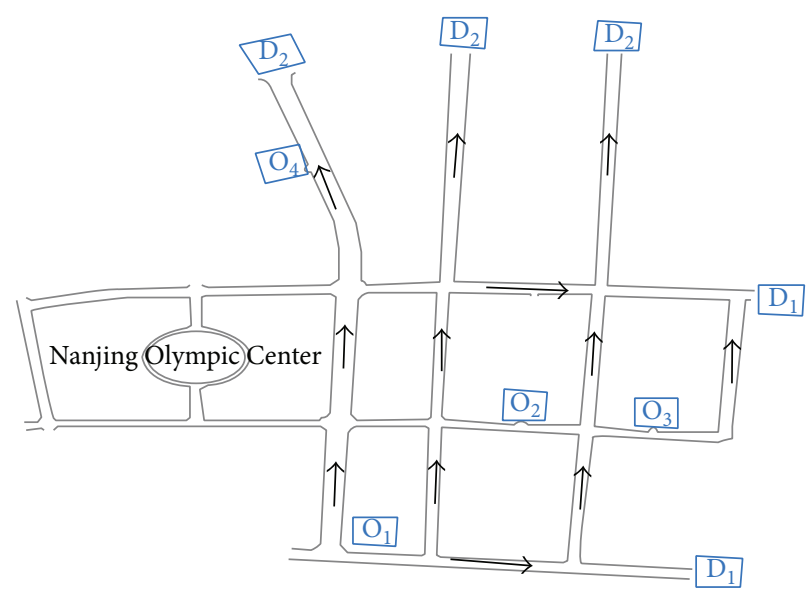

(a)

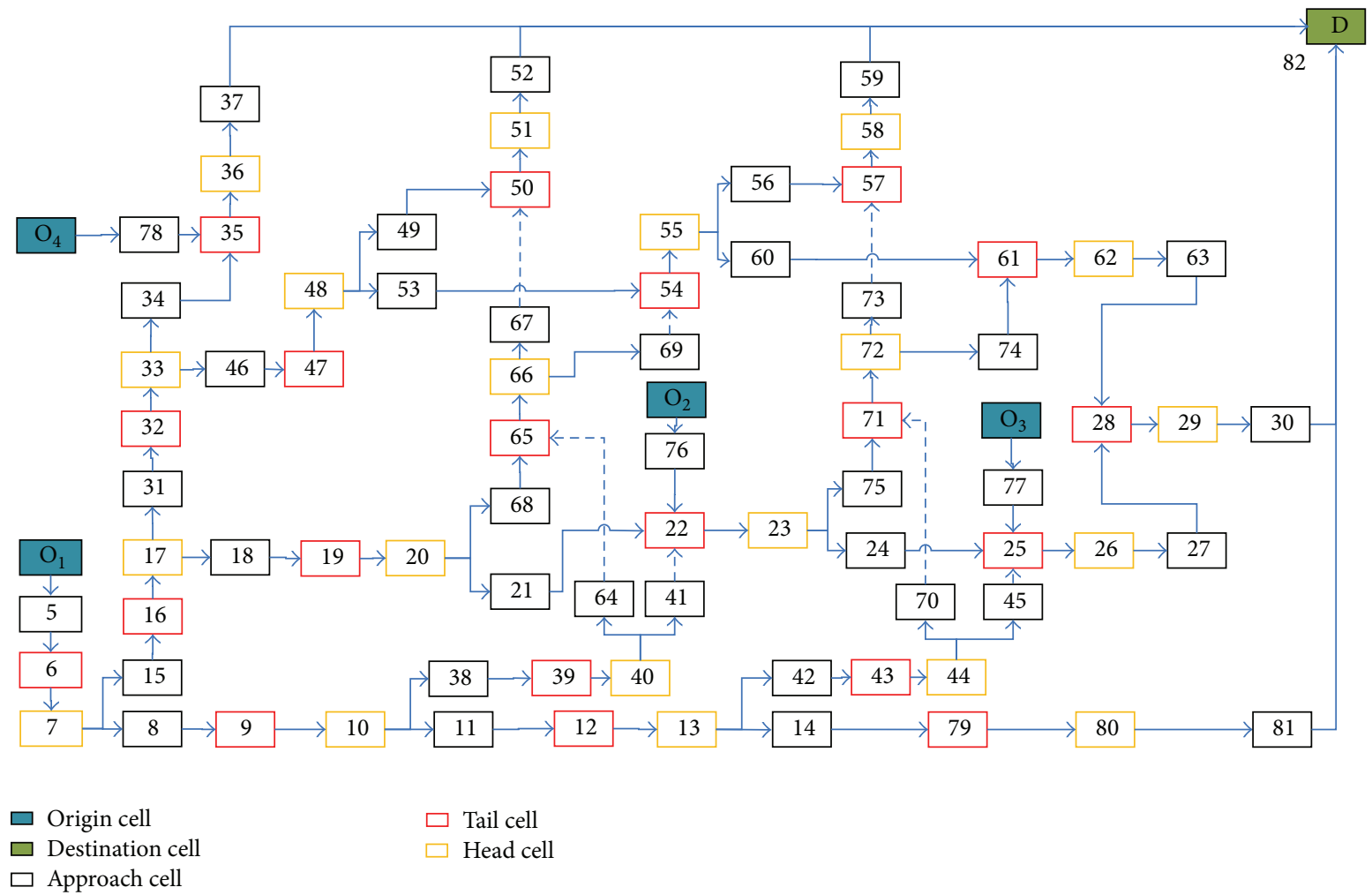

(b)

FIGURE 3: Evacuation road network: (a) original road network; (b) corresponding cell representation.

We implement route-choice SP survey under aforementioned evacuation-scenario assumption. Using the survey data, specify the utility coefficients by maximum likelihood method. The probability of a route $r \in I_{i}$ chosen by evacuees at interval $\tau$ is formulated as follows:

$p_{i, r}^{\tau}=\left(\left(\sum_{c \in C_{r}} w_{c, r} n_{c, r}\right)^{0.04} \exp \left(-2.2 L_{r}-1.1 D_{r}^{\tau}-0.8 R_{r}^{\tau}\right)\right)$

$$
\begin{aligned}
\times\left(\sum_{h \in I_{i}}(\right. & \left(\sum_{d \in C_{h}} w_{d, h} n_{d, h}\right)^{0.04} \\
& \left.\left.\times \exp \left(-2.2 L_{h}-1.1 D_{h}^{\tau}-0.8 R_{h}^{\tau}\right)\right)\right)^{-1} .
\end{aligned}
$$

Only one information factor is allowed to be selected as the decision variable at each origin per information-updating 
TABLE 1: Traffic demand and characteristics of cell.

\begin{tabular}{lc}
\hline Index type & Assigned number \\
\hline Total demand (which would depart uniformly & $450 \mathrm{veh}$ \\
in 15 mins) of each origin & $6 \mathrm{~s}$ \\
Length of (loading) time interval & $180 \mathrm{~s}$ \\
Length of information-updating time interval & $10 \mathrm{~m} / \mathrm{s}$ \\
Free-flow speed & 6 basic cells \\
Length of head and tail cell & $6 \mathrm{veh}$ \\
Maximum occupancy of approach cell 14, 44 & $12 \mathrm{veh}$ \\
Maximum occupancy of approach cell 42, 75 & $9 \mathrm{veh}$ \\
Maximum occupancy of other approach cells & $108 \mathrm{veh}$ \\
Maximum occupancy of head and tail cells & $1 \mathrm{veh}$ \\
Capacity of approach cell 14, 24 per time & \\
interval & $5 \mathrm{veh}$ \\
Capacity of approach cell 42, 75 per time & \\
interval & $3 \mathrm{veh}$ \\
Capacity of other approach cells per time & $6 \mathrm{veh}$ \\
interval & \\
Capacity of head and tail cells per time interval &
\end{tabular}

interval. The route feature and variable range are the criteria in this case.

(i) The route of shortest length from each origin cell is chosen as the route for decision variable setting, because more variations occurring in the shortest length route can create a broader range as the candidate of the strategic information variable.

(ii) When the coefficients are close, the information factors with broader ranges are chosen as the decision variable because they allow adjusting route-choice probability greatly.

Once the decision variable is determined, other information variables could be assigned with real-time values or the other corresponding value depending on the relation among the information values.

In this case, for each origin cell, the delay time of shortestlength route is set as the decision variable according to the criterion description. The delays in other routes are realtime values obtained from the flow simulation, which could occur most probably in the near-future traffic conditions. The disseminated delay-time reliabilities are determined based on the near-future delay-time distribution. Each probability of the disseminated delay time is assigned to its reliability in the application. To be convenient, we assume the delay time obeys the normal distribution with the mean of the real-time delay $a_{r}^{\tau}$ and standard deviation of $b$. The real-time delay is undoubtedly the most probable value, which is in accord with the previous assumption. The disseminated delay-time reliability can be represented as follows:

$$
R_{r}^{\tau}=f\left(\frac{D_{r}^{\tau}-a_{r}^{\tau}}{b}\right) \cdot\left(\frac{\widetilde{U}-\tilde{L}}{\vartheta}\right)
$$

where the function $f(\cdot)$ is the probability density function of standard normal distribution. $\vartheta$ is the divided number of delay-time range, which is set as 10 in the following application. It is used to ensure that the reliability is roughly equal to probabilistic density multiplying unit range of the divided delay time. Besides, $b$ is set to be 1 ; the range of strategic delay time $[\widetilde{L}, \widetilde{U}]$ is set to be $[0,10]$ mins.

3.2. Test. The advantage of the application of the D-EDO in creating an optimal traffic information strategy is verified. This strategy outperforming reactive information control is indicated. In addition, the influence of information-updating frequency and demand level are analyzed.

3.2.1. Effectiveness of the D-EDO. $\varepsilon=1$ is set as the end criteria of D-EDO algorithm. The corresponding solution convergence of the D-EDO is shown in Figure 4 . When EDO algorithm completes an updating of all the variables per loop, we use the current minimal boundary solution of the acquired two boundary solutions as the initial point to obtain the corresponding F-W result. The minimal boundary solution convergence of EDO showed that the single EDO method may not achieve the minimal TSTT even compared with the historical iterations. However, the FW can contribute to obtaining lower TSTT when taking the intermediate EDO minimal boundary solution as the initial solution. Varied initial solution can achieve varied result using F-W; thus, the EDO combined with F-W can complement each other. The minimal TSTT selected from all the F-W solutions is 122,500 veh time intervals, with the variable solution listed in Table 3.

We use the genetic algorithm (GA) as another heuristic algorithm to compare with D-EDO. The GA is configured to have an initial population of 40 , a crossover probability of 0.9 , and mutation probability of 0.1 . We set the maximal allowed tolerance generation number of unchanged fitness to be 5 , which is the end criterion.

Seeing from Figure 4, the D-EDO achieves a better solution compared with the GA. The primary advantage of DEDO method over the GA method is that the accuracy result is obtained. Although the GA converges with few generations, the calculation time is not saved significantly with nearly 40 times of flow simulation in each generation. To avoid the premature convergence, some improved GA (such as micro GA) may be available in finding the optimal solution, but the calculation time will also increase. Therefore, we use the DEDO algorithm to analyze the following problems.

\subsubsection{Advantage of the Proactive Traffic Information Com-} pared with Reactive Traffic Information. If the real-time delays are set as the information, the ISTT would be 138,212 veh time intervals, which is higher than that using the proactive values. The real-time delay information has not considered the route-choice feedback influenced by congestion. However, the PTIC can mitigate congestion effectively and the eventual evacuation network will be more safe and reliable compared to the result of reaction traffic information control. 
TABLE 2: Candidate route set and the commonality factor of each route.

\begin{tabular}{ccc}
\hline Origin code & Candidate route set & $\sum_{c \in C_{r} w_{c, r} n_{c, r}}$ \\
\hline & $O_{1} \rightarrow 7 \rightarrow 10 \rightarrow 13 \rightarrow 80 \rightarrow D$ & $23 / 20 ;$ \\
1 & $O_{1} \rightarrow 7 \rightarrow 17 \rightarrow 33 \rightarrow 36 \rightarrow D$ & $3 / 4 ;$ \\
& $O_{1} \rightarrow 7 \rightarrow 10 \rightarrow 40 \rightarrow 66 \rightarrow 51 \rightarrow D$ & $23 / 20$ \\
\hline & $O_{2} \rightarrow 23 \rightarrow 26 \rightarrow 29 \rightarrow D$ & $5 / 3 ;$ \\
$O_{2} \rightarrow 23 \rightarrow 72 \rightarrow 58 \rightarrow D$ & $1 ;$ \\
& $O_{2} \rightarrow 23 \rightarrow 72 \rightarrow 62 \rightarrow 29 \rightarrow D$ & $7 / 3$ \\
\hline
\end{tabular}

Table 3: Proactive traffic information solution/mins.

\begin{tabular}{lccccc}
\hline Time period of information updating & 1 & 2 & 3 & 4 & 5 \\
\hline $\begin{array}{l}\text { Published delay of route } \\
O_{1} \rightarrow 7 \rightarrow 10 \rightarrow 13 \rightarrow 80 \rightarrow D \text { at cell 1 }\end{array}$ & 5.9 & 1.1 & 10 & 2.3 & 8.5 \\
Published delay of route & 2.8 & 10 & 4.1 & 8.2 & 0 \\
$O_{2} \rightarrow 23 \rightarrow 26 \rightarrow 29 \rightarrow D$ at cell 6 & & & & & \\
\hline
\end{tabular}

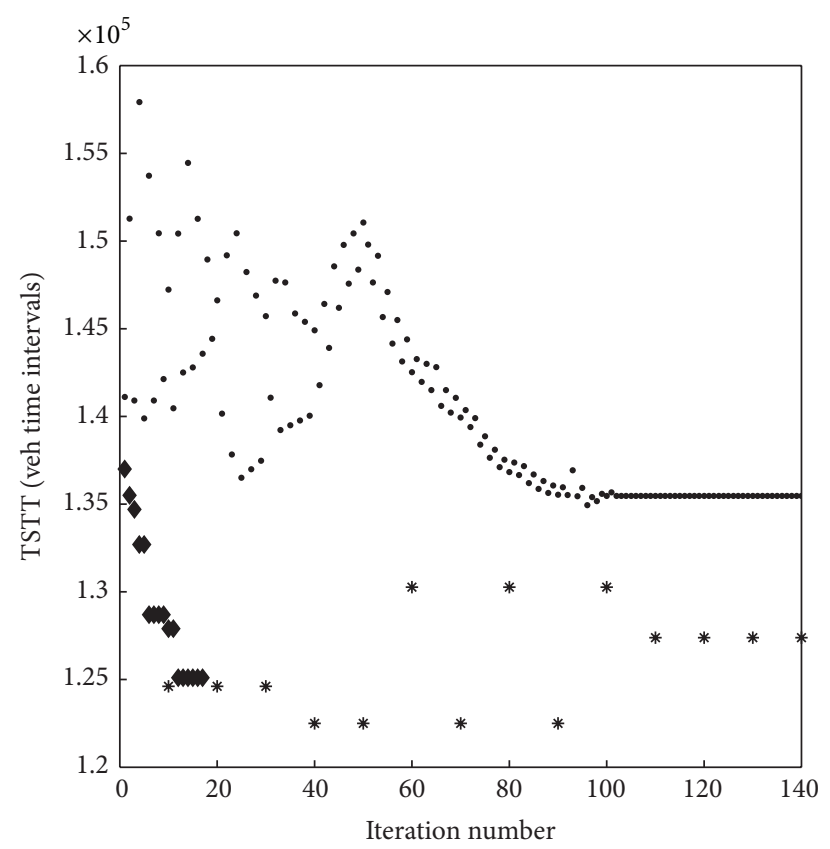

- Minimal boundary solution at each EDO iteration within D-EDO

* F-W solution within D-EDO

$\checkmark$ GA solution

FIGURE 4: Convergence comparison about the solutions between DEDO and GA.

3.2.3. Correlation of Information-Updating Frequency and TSTT. The needed time interval per information-updating gap at each origin cell is changed from 10 to 50; then, we calculate TSTT for each information-updating frequency combination during the change process. The results are listed in Figure 5. Although few unusual situations exist where the increase of information-updating frequency creates higher TSTT, the majority of TSTT trends can roughly show that the higher frequency has more opportunity to create a lower
TSTT. This implies that an increase in frequency has a positive effect on evacuation efficiency improvement.

Before the recommendation of information-updating frequency, we classify the frequency group into three types according to the closeness of TSTT. High frequency is normally recommended; however, some inflection points of frequency may be better choices. There are two reasons why overhigh frequency is not a better choice. First, it is lack of room for evacuation efficiency improvement in high frequency range as Figure 5 shows: the TSTT improves rapidly at the low frequency range (group 3), while it improves more slowly at a high frequency range (group 1). Second, it is subject to the constraints of information collection technology, which does not allow a short updating time. Therefore, the frequency range in group 2 may be a recommendation for decision makers.

3.2.4. Correlation of Demand Matrix Multiplication Factor and Average Cost per Evacuee. As shown in Figure 6, when the demand matrix multiplication factor is below 1 , average cost per evacuee increases slowly. When the factor exceeds 1 , average cost per evacuee increases dramatically with demand matrix multiplication factor and the rate of change is almost the same. This indicates that network operation using PTIC may be not effective in the high evacuation demand range. We can set the demand assumed in this case as the boundary in which the PTIC works. When the average cost per evacuee increases sharply, we should also depend on other methods such as the design of contraflow lanes $[13,14]$ and evacuation schedule [15] to control the evacuation congestion.

\section{Conclusion and Discussion}

Considering the uncertainty of the evacuation flows, a PTIC method for network evacuation is constructed, wherein the information value is selected from a range instead of adopting real-time value directly. With the preparedness of route-choice utility investigation, we adopt the one-shot stochastic dynamic traffic assignment as the constraint and set the minimal total system travel time as the objective to construct the framework model, wherein congestion cost is embedded to mitigate oversaturated traffic. D-EDO is proposed to be the algorithm to solve the proactive traffic information. Compared to other network design methods, DEDO can achieve a more accurate result. The result shows that PTIC can obtain lower objective value than reactive traffic information control. 


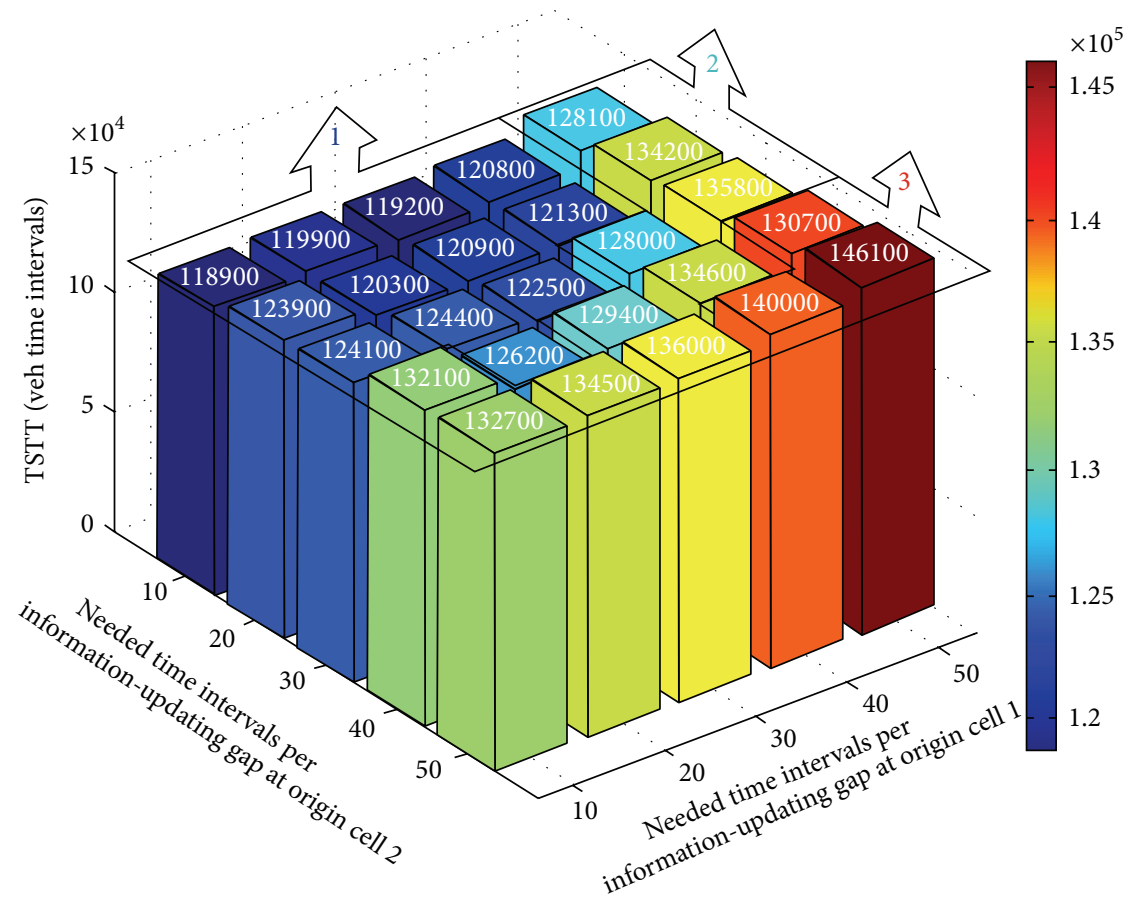

FIGURE 5: Relation of information-updating frequency and TSTT.

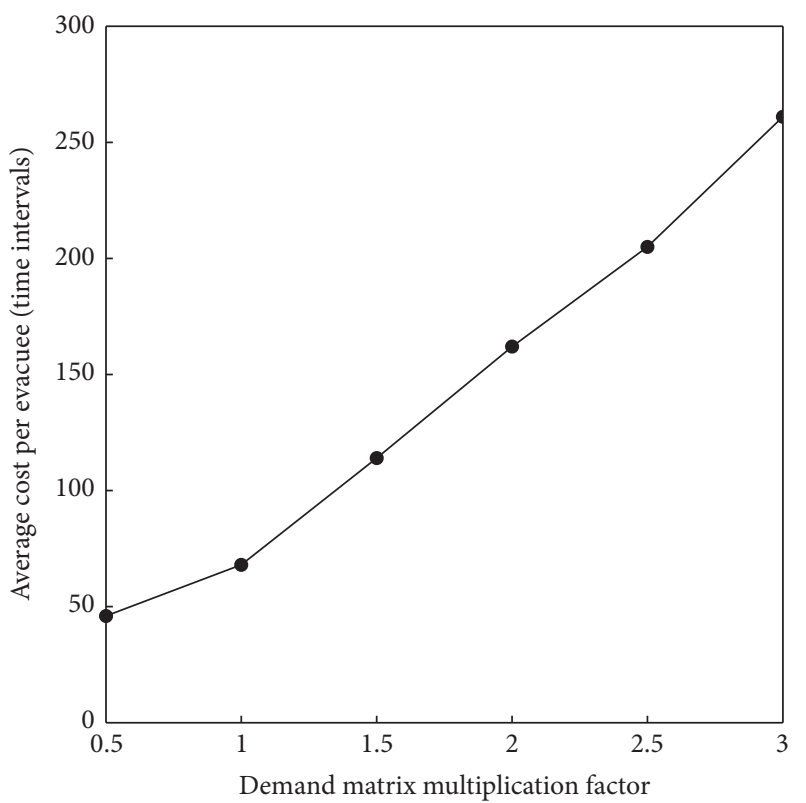

FIGURE 6: Change of average cost per evacuee with demand matrix multiplication factor.

We extend the study to the influence of informationupdating frequency and demand level. An increase in information-updating frequency is welcome. However, the frequency of inflection point is advised to be chosen as the application value instead of high technology-cost frequency.
With respect to the demand influence, average cost per evacuee increases rapidly in higher demand range. Thus, the application of PTIC is effective under a specific demand level.

Three points should be noticed in the future research. (1) The evacuation of excessive demands is beyond the application scope of this method. How to combine PTIC and other traffic management measures to deal with excessive demands is a significant study direction. (2) The candidate route set of the case is generated a priori, because dynamically updating the routes is a challenge. The technology of column generation applied in static traffic assignment or numberlimited dynamic routes proposed by Chiu and Mirchandani [4] may be useful. (3) Before making this method a promising solution to meet the emergency evacuation need, the distribution function of the fluctuated delay time should be investigated with field delay data.

\section{Conflict of Interests}

The author declares that there is no conflict of interests regarding the publication of this paper.

\section{Acknowledgments}

This research is supported by the National Natural Science Foundation of China (no. 51408321). The author appreciates the Wisconsin Traffic Operations and Safety Laboratory. The author appreciates Professor Gang Ren, Professor Bin Ran, Professor Haixu Liu, Dr. Yang Cheng, and Dr. Peter J. Jin for their professional advices. 


\section{References}

[1] G. A. Davis, "Exact local solution of the continuous network design problem via stochastic user equilibrium assignment," Transportation Research Part B, vol. 28, no. 1, pp. 61-75, 1994.

[2] E. Cascetta, M. Gallo, and B. Montella, "Models and algorithms for the optimization of signal settings on urban networks with stochastic assignment models," Annals of Operations Research, vol. 144, pp. 301-328, 2006.

[3] C. Xie and M. A. Turnquist, "Integrated evacuation network optimization and emergency vehicle assignment," Transportation Research Record: Journal of the Transportation Research Board, no. 2091, pp. 79-90, 2009.

[4] Y.-C. Chiu and P. B. Mirchandani, "Online behavior-robust feedback information routing strategy for mass evacuation," IEEE Transactions on Intelligent Transportation Systems, vol. 9, no. 2, pp. 264-274, 2008.

[5] A. Paz and S. Peeta, "Behavior-consistent real-time traffic routing under information provision," Transportation Research Part C: Emerging Technologies, vol. 17, no. 6, pp. 642-661, 2009.

[6] C. Suwansirikul, T. L. Friesz, and R. L. Tobin, "Equilibrium decomposed optimization: a heuristic for the continuous equilibrium network design problem," Transportation Science, vol. 21, no. 4, pp. 254-263, 1987.

[7] A. H. F. Chow and H. K. Lo, "Sensitivity analysis of signal control with physical queuing: delay derivatives and an application," Transportation Research Part B: Methodological, vol. 41, no. 4, pp. 462-477, 2007.

[8] Y. Yin, "Genetic-algorithms-based approach for bilevel programming models," Journal of Transportation Engineering, vol. 126, no. 2, pp. 115-119, 2000.

[9] M. Wardman, P. W. Bonsall, and J. D. Shires, "Driver response to variable message signs: a stated preference investigation," Transportation Research Part C: Emerging Technologies, vol. 5, no. 6, pp. 389-405, 1997.

[10] H. Hua, Y.-F. Gao, and X.-G. Yang, "Probabilistic traffic forecast method based on comprehensive transport information platform," Journal of Traffic and Transportation Engineering, vol. 9, no. 3, pp. 122-126, 2009.

[11] E. Cascetta, A. Nuzzolo, F. Russo, and A. Vitetta, "A modified logit route choice model overcoming path overlapping problems: specification and some calibration results for interurban networks," in Proceedings of the 13th International Symposium on Transportation and Traffic Theory, Lyon, France, 1996.

[12] C. F. Daganzo, "The cell transmission model: a dynamic representation of highway traffic consistent with the hydrodynamic theory," Transportation Research Part B: Methodological, vol. 28, no. 4, pp. 269-287, 1994.

[13] B. Wolshon, "One-way-out: contraflow freeway operation for hurricane evacuation," Natural Hazards Review, vol. 2, no. 3, pp. 105-112, 2001.

[14] H. Tuydes and A. Ziliaskopoulos, "Tabu-based heuristic approach for optimization of network evacuation contraflow," Transportation Research Record, vol. 1964, pp. 157-168, 2006.

[15] H. Sbayti and H. S. Mahmassani, "Optimal scheduling of evacuation operations," Transportation Research Record: Journal of the Transportation Research Board, vol. 1964, pp. 238-246, 2006. 


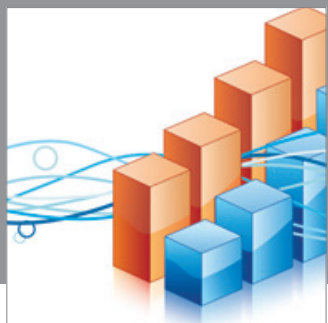

Advances in

Operations Research

mansans

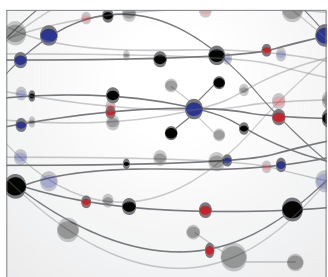

The Scientific World Journal
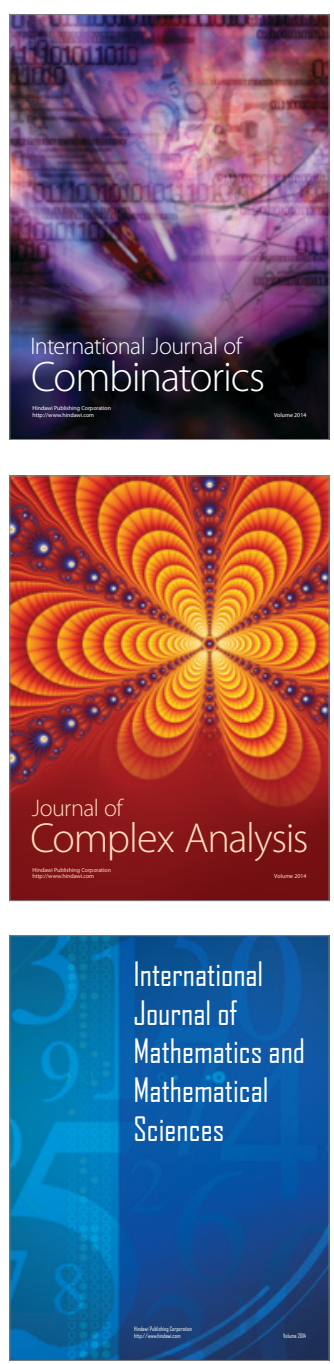
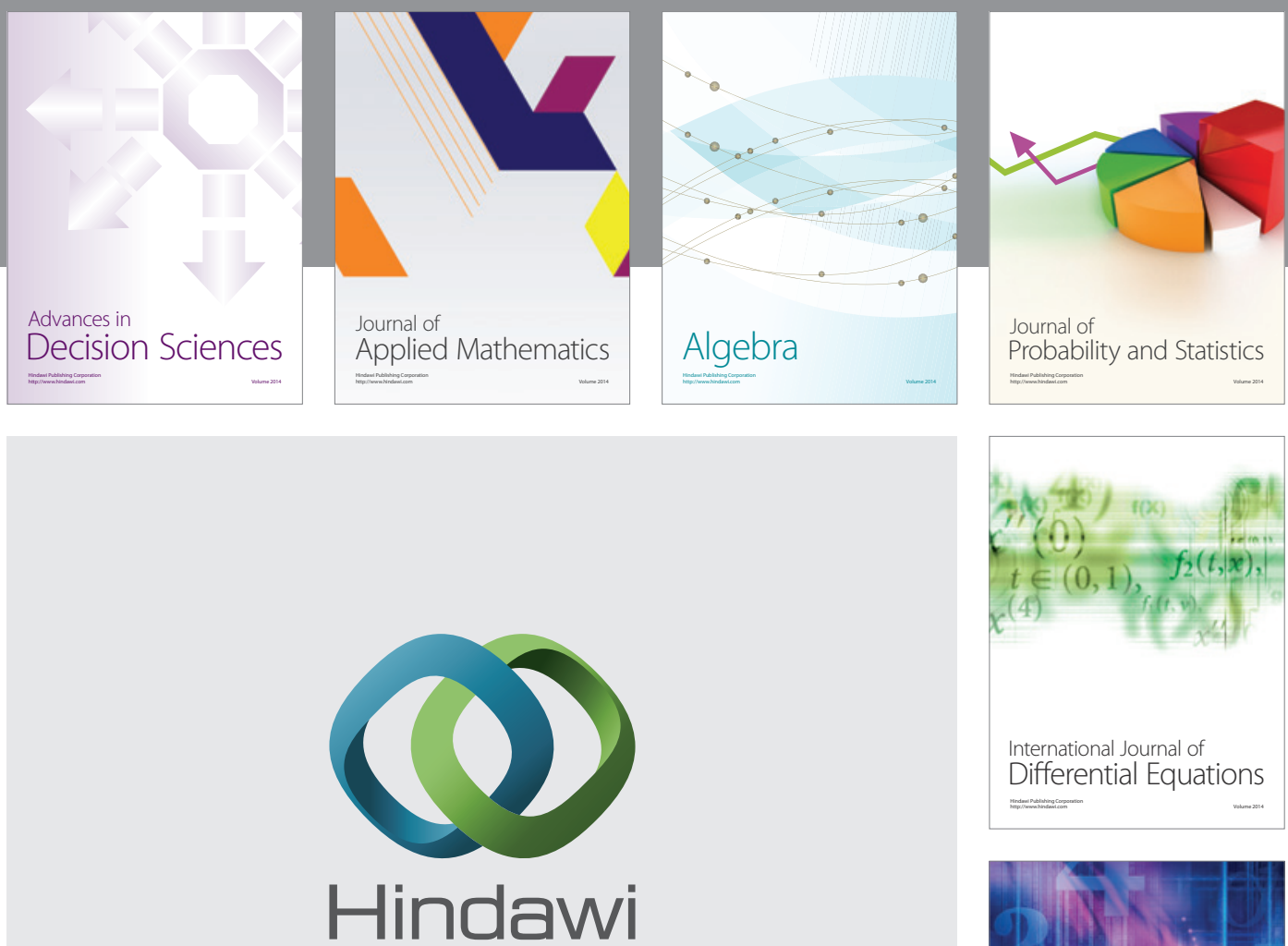

Submit your manuscripts at http://www.hindawi.com
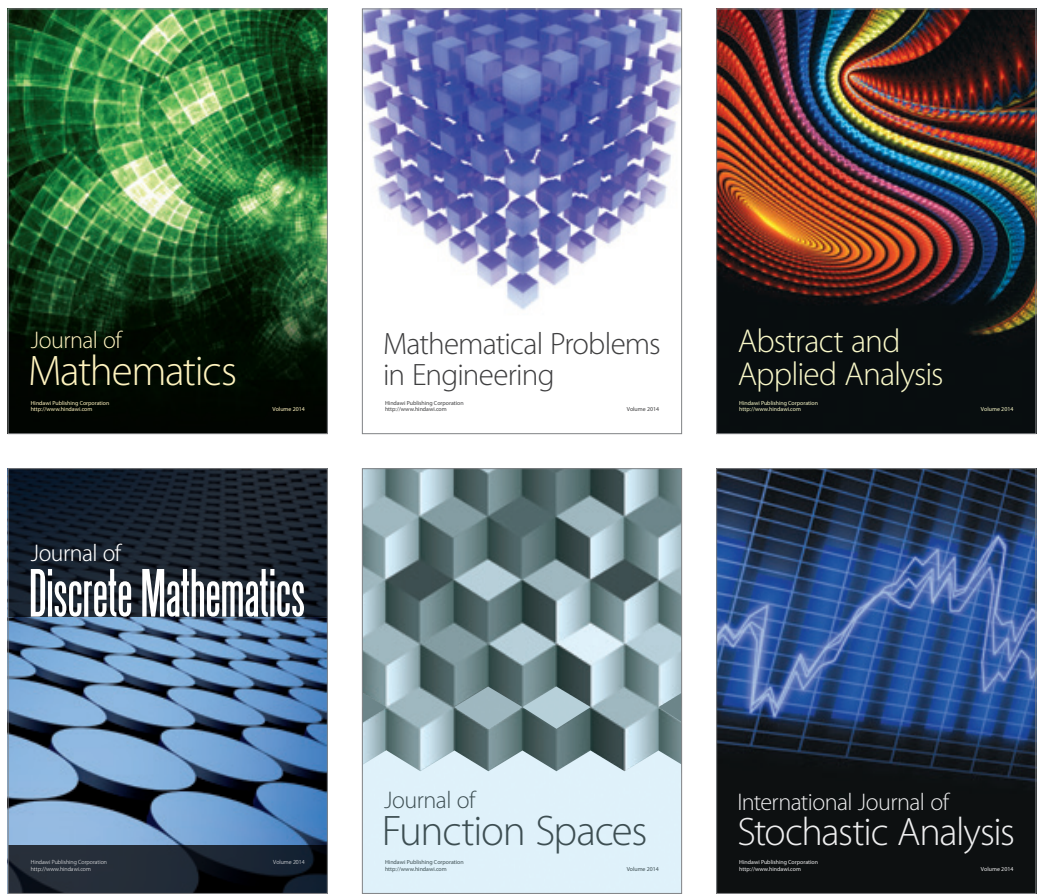

Journal of

Function Spaces

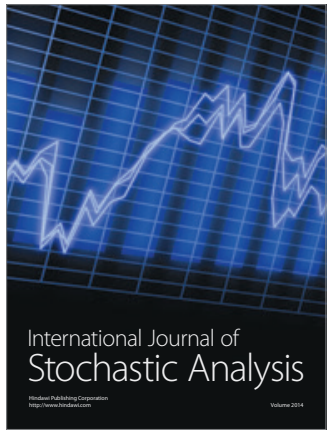

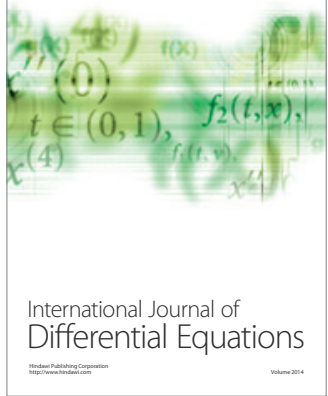
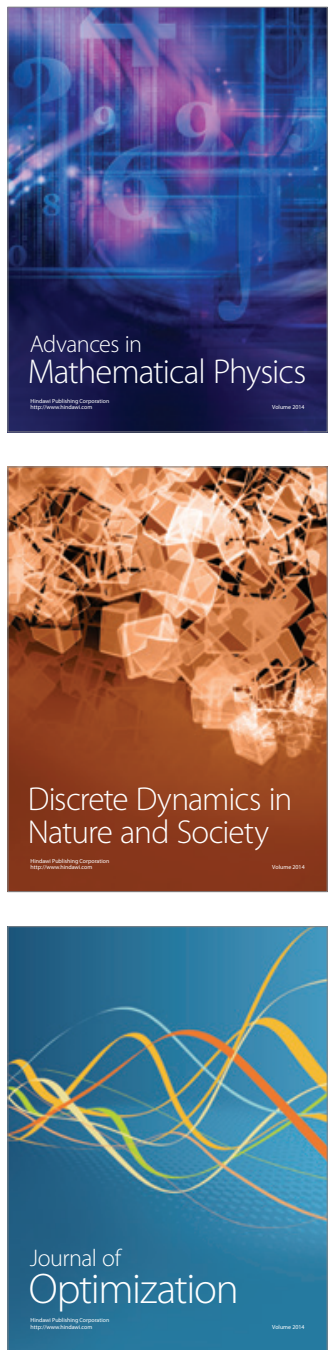